Urologe 2019.58:931-932

https://doi.org/10.1007/s00120-019-0975-5

Online publiziert: 30 . Juli 2019

(c) Springer Medizin Verlag GmbH, ein Teil von Springer Nature 2019

\section{Julia Skutella}

Klinik für Urologie, Andrologie und Kinderurologie, Kliniken Nordoberpfalz AG, Weiden, Deutschland

\title{
Nachwuchsmangel als Chance und Herausforderung für die Weiterentwicklung der Urologie
}

„Urologie - das hat auch etwas mit den Nieren zu tun“. Das war die Antwort meines Mannes, die er meist etwas verschämt gab, als er zu Beginn meiner ärztlichen Tätigkeit von Bekannten und Verwandten gefragt wurde, für welche Fachrichtung ich mich entschieden habe. Die Reaktionen darauf waren sehr unterschiedlich und reichten von spontan vorgetragenen Penis-Witzen bis zu Anmerkungen, dass man doch einen Dermatologen, der einem die Besenreiser lasert, besser brauchen könnte.

Meinen ersten Kontakt mit dem Fach Urologie hatte ich noch vor dem Studium. Ich absolvierte das komplette Pflegepraktikum auf einer urologischen Station und zwar in der Abteilung, in der ich jetzt als Arzt tätig bin. Das Medizinstudium absolvierte ich in München und Regensburg eigentlich mit dem festen Plan Internist zu werden. Erst im höheren Semester, mit Durchlaufen der „kleinen“ Fächer, entdeckte ich die Urologie für mich. Es folgten Famulatur und PJ-Wahltertial. 2013 begann ich als Assistenzarzt in der urologischen Abteilung des Klinikum Weiden. Im April 2018 legte ich die Facharztprüfung ab. Kurz darauf übernahm ich für 2 Monate eine Praxisvertretung und durfte so auch diesen Sektor und somit einen anderen Tätigkeitsbereich des Urologen kennenlernen.

Arzt zu sein, ist für mich, trotz der oft hohen Arbeitsbelastung und damit verbundenen Einbußen im Privatleben, die befriedigendste Tätigkeit, die ich mir vorstellen kann. Das Fach Urologie begeistert mich täglich trotz des überschaubaren Organsystems mit seiner enorm umfangreichen und damit auch herausfordernden Vielfalt an diagnostischen und therapeutischen Möglichkeiten. Gerade bei der Behandlung von tabuisierten Erkrankungen wie Inkontinenz, Erektionsstörung usw. bedarf es einer besonderen Offenheit bei der Patientenbetreuung, die das Fach Urologie für mich menschlich besonders reizvoll macht.

Ich arbeite seit 6 Jahren als Arzt, nur eine kleine Spanne im Hinblick auf ein immer länger werdendes Arbeitsleben. Trotz des begrenzten Zeitraums sind seit Beginn meiner Tätigkeit einige Veränderungen deutlich spürbar.

Als wachsendes Problem, das wir mit vielen peripheren Häusern teilen, sehe ich den zunehmenden Personalmangel. Die Landflucht, die zu einem Fachkräftemangel im ländlichen Raum führt, betrifft meinen Arbeitgeber genauso wie unsere urologische Abteilung. Hatte ich bei meiner Einstellung noch das Gefühl gehabt, froh sein zu müssen, eine freie Stelle ergattert zu haben, scheint mir bereits in der begrenzten Zeit meiner ärztlichen Tätigkeit ein Wandel stattgefunden zu haben, der die Auswahlmöglichkeiten und Freiheiten von heutigen Studienabgängern betrifft. Mehrfach höre ich im Bekanntenkreis, dass nach Studienabschluss noch eine längere Reise unternommen oder noch Zeit für das Feilen an der Doktorarbeit genommen wird kein Problem, die Stelle wartet. Nicht die Bewerber, sondern die Kliniken durchlaufen ein Assessment und überschlagen sich beim Buhlen um den Nachwuchs. Unsere Klinik hat auf den Mangel reagiert und befürwortet bei geeigneten $\mathrm{Be}$ werbern auch die Einstellung über den Stellenplan - vor ein paar Jahren schien das noch undenkbar.
Personalmangel bedeutet automatisch eine höhere Arbeits- und Dienstbelastung. Bewerbungen aus dem Ausland werden deswegen für uns immer wichtiger. Waren es früher speziell in unserer Region Kollegen aus den osteuropäischen Nachbarländern, wie z.B. Tschechien, nimmt diese Ressource zunehmend ab, da der Anreiz für eine Auswanderung nach Deutschland bei steigenden Löhnen in den Heimatländern sinkt. Dafür steigt die Bewerberzahl aus dem Nahen Osten und Russland. Bei unterschiedlichem Ausbildungsstand sehe ich eine besondere Herausforderung darin, diese Kollegen strukturiert auszubilden und in unser System zu integrieren.

Die Gender- und Paritätsdiskussion ist momentan brandaktuell, so macht die viel diskutierte Forderung nach einer Frauenquote auch vor dem Gesundheitswesen nicht halt. Fakt ist, dass die Medizin mit einem zunehmenden Frauenanteil der Medizinstudierenden immer weiblicher wird, selten aber „an der Spitze“ in Form von mit Frauen besetzten Führungspositionen. Gerade in der Urologie mit im Vergleich zu anderen Fachabteilungen geringem Frauenanteil scheint mir eine Diskussion über Frauen in der Urologie und Parität als weiteres aktuelles Problem angebracht. Früher ging ich immer davon aus, dass weibliche und männliche Ärzte vielleicht nicht gleich, aber durchaus gleichberechtigt sind. Mittlerweile sehe ich es anders. „Frau sein“ verbinde ich mittlerweile mit deutlich schlechteren Karrierechancen besonders in einer operativen Abteilung. Karrierehindernis Nummer eins für Ärztinnen bleibt die Schwangerschaft - ist sie doch weiterhin in der Regel mit 
einem Aussetzen der operativen Tätigkeit verbunden. Monate, die im OP und beim Erlangen der Weiterbildung eine Ewigkeit darstellen. Die Novellierung des Mutterschutzgesetzes von 2018 stellt dabei leider, wie zunächst von vielen Ärztinnen gehofft, keine Verbesserung dar, so fällt doch die individuelle Gefährdungsbeurteilung für den OP-Bereich der meisten Häuser negativ aus, sodass Schwangeren der Zugang zu operativen Tätigkeiten verwehrt bleibt. Es ist Alltag in unserer Abteilung, wie wohl in vielen anderen Kliniken auch, dass manche Kolleginnen eine Schwangerschaft möglichst lange geheim halten, um noch möglichst viel operative Erfahrung zu sammeln. Bei uns bedeutet dies auch eine weitere Teilnahme an den anstrengenden Vordergrunddiensten, die in der Regel am Ende der limitierende Faktor ist und die eine Kollegin früher, die andere später dazu bringt, aus der Dienstgenauso wie aus der OP-Rotation auszuscheiden. Dies bedeutet wiederum eine höhere Dienst- und Arbeitsbelastung für die Kollegen, sodass es nicht unüblich ist, sich bei Verkünden einer Schwangerschaft bei jedem Kollegen einzeln zu entschuldigen. Nach Schwangerschaft und Elternzeit bedeutet eine Rückkehr in Teilzeit ebenfalls eine erneute Einbuße bei der Weiterbildung, so berichten mir Kolleginnen ihre erlangten Fähigkeiten in Teilzeit gerade so erhalten zu können, aber nur schwer etwas neues dazu zu lernen. Gerade in kleineren Häusern mit geringer Personalzahl kann auf die Bedürfnisse von Müttern und jungen Familien oft nur schlecht eingegangen werden, so sind gerade die Vordergrunddienste für manche Kollegin der Grund, die Klinik zu verlassen und ggf. in eine Anstellung in der Praxis zu wechseln. Manche Wegbegleiterin wechselte sogar nach der Facharztprüfung in einen familienfreundlicheren Fachbereich. Natürlich befindet sich unsere Gesellschaft im Wandel. Mittlerweile gibt es auch Väter, die mehr als die heutzutage standardmäßigen 2 Monate Elternzeit in Anspruch nehmen und sich somit deutlich mehr als unsere Elterngeneration bei der Kindererziehung und -betreuung einbringen, und nicht für jede Frau besteht das Lebensziel im Zeugen vie- ler Kinder und Teilzeitarbeit. Trotzdem werden gewisse Rollenmuster in unserer Gesellschaft nach wie vor gelebt. Arbeitgeber können selbst bei Frauen, für die Familienplanung (noch) kein Thema ist, nicht wissen, ob sie in Zukunft durch Schwangerschaft und Elternzeit ausfallen und sich auf Dauer familienfreundlichere Stellen suchen. Somit ist die Anstellung von Frauen mit Risiken verbunden und macht sie gerade im operativen Bereich für mögliche Förderungen unattraktiver als ihre männlichen Kollegen.

Ich gehe davon aus, dass im Jahr 2025 (in 6 Jahren) - also genau in der Verdopplung der Zeit, die ich bisher als Arzt in der Urologie verbracht habe, die Arbeitsbelastung aufgrund des demographischen Wandels allgemein und im Besonderen in der Peripherie, sowie der langfristig abnehmenden Zahl von Ärzten deutlich zunehmen wird. Die Konkurrenz im Werben um den immer weniger werdenden ärztlichen Nachwuchs wird weiter steigen.

Um diesem Wandel zu begegnen, müssen unsre strukturellen Arbeitsbedingungen angepasst werden. Bestrebungen ärztliche Tätigkeiten in Kliniken auf nicht-ärztliches Personal wie z.B. physician assistents zu delegieren, sehe ich zwar kritisch, aber als unumgänglich an. Des Weiteren muss eine Vereinfachung von Arbeitsprozessen z.B. durch Nutzen der Digitalisierung erreicht werden.

Dem Nachwuchsmangel müssen wir entgegentreten, in dem wir Studierende und Studienabgänger für das Fach Urologie begeistern und rekrutieren. Bietet die Urologie doch optimale Grundvoraussetzungen, was die Vielfalt des Faches angeht, muss die Attraktivität durch eine strukturierte Weiterbildung weiter gesteigert werden.

Bei wachsendem Frauenanteil der Medizinstudierenden wäre es dumm, die Ressource „Frau“ nicht zu nutzen. Das heißt die Urologie muss, genau wie die anderen chirurgischen Fächer, attraktiver für Frauen werden. Der Schlüssel wird auch hier die strukturierte Weiterbildung sein, sowie das Schaffen von Aufstiegsmöglichkeiten und flexibleren Arbeitsmodellen. Hier sind gerade Klinken gefragt, ärztliche Kolleginnen zu halten und nicht an eine familienfreundlichere An- stellung in der Praxis zu „,verlieren“. Ich habe vor kurzem eine leitende Oberärztin in der Gynäkologie kennengelernt, die sich ihren Posten mit einer Kollegin teilt. Ich denke, dass derartige individuelle Arbeits- und Stellenkonzepte gerade in Anbetracht einer weniger werdenden $\mathrm{Zahl}$ von Generalisten selbst im Fach Urologie Schule machen könnten.

Ärztinnen müssen selbst den offen und ehrlichen Dialog mit ihren Arbeitgebern bzgl. Familienplaung suchen, um gemeinsam individuelle Weiterbildungsund Förderungskonzepte zu entwickeln.

Mein Fazit: Die Urologie ist ein großartiges Fach, das ich immer wieder wählen würde. Herausforderung wird in den nächsten Jahren u.a. der Nachwuchsmangel darstellen. Dieser könnte eine Chance für einen - wie ich hoffe - positiven Wandel des ärztlichen Arbeitens darstellen, fordern doch die nachfolgenden Generationen deutlich mehr eine ausgeglichenere Work-Life-Balance als ihre Vorgänger. Allerdings muss, um diesen zu erreichen, gerade in Anbetracht der wachsenden Arbeitsbelastung eine Weiterentwicklung der strukturellen Arbeitsbedingungen erfolgen. Die Urologie hat als innovativer Fachbereich das Potenzial, diese Herausforderungen zu meistern.

\section{Korrespondenzadresse

Julia Skutella
Klinik für Urologie, Andrologie
und Kinderurologie, Kliniken
Nordoberpfalz AG
Söllnerstr. 16,92637 Weiden,
Deutschland
julia.skutella@kliniken-
nordoberpfalz.ag

Interessenkonflikt. J. Skutella gibt an, dass kein Interessenkonflikt besteht. 